\title{
Genetic/metabolic effect of iron metabolism and rare anemias
}

\section{Clara Camaschella}

\author{
Vita-Salute University and San Raffaele Scientific Institute, Milan, Italy
}

\begin{abstract}
Advances in iron metabolism have allowed a novel classification of iron disorders and to identify previously unknown diseases. These disorders include genetic iron overload (hemochromatosis) and inherited iron-related anemias, in some cases accompanied by iron overload. Rare inherited anemias may affect the hepcidin pathway, iron absorption, transport, utilization and recycling. Among the genetic iron-related anemias the most common form is likely the iron-refractory iron-deficiency anemia (IRIDA), due to mutations of the hepcidin inhibitor TMPRSS6 encoding the serine protease matriptase-2. IRIDA is characterized by hepcidin up-regulation, decrease iron absorption and macrophage recycling and by microcytic-hypochromic anemia, unresponsive to oral iron. High serum hepcidin levels may suggest the diagnosis, which requires demonstrating the causal TMPRSS6 mutations by gene sequencing. Other rare microcytic hypochromic anemias associated with defects of iron transport-uptake are the rare hypotransferrinemia, and DMT1 and STEAP3 mutations. The degree of anemia is variable and accompanied by secondary iron overload even in the absence of blood transfusions. This is due to the iron-deficient or expanded erythropoiesis that inhibits hepcidin transcription, increases iron absorption, through the erythroid regulator, as in untransfused beta-thalassemia. Sideroblastic anemias are due to decreased mitochondrial iron utilization for heme or sulfur cluster synthesis. Their diagnosis requires demonstrating ringed sideroblasts by Perl's staining of the bone marrow smears. The commonest X-linked form is due to deltaamino-levulinic-synthase-2-acid (ALAS2) mutations. The recessive, more severe form, affects SLC25A38, which encodes a potential mitochondrial importer of glycine, an amino acid essential for ALA syn-
\end{abstract}

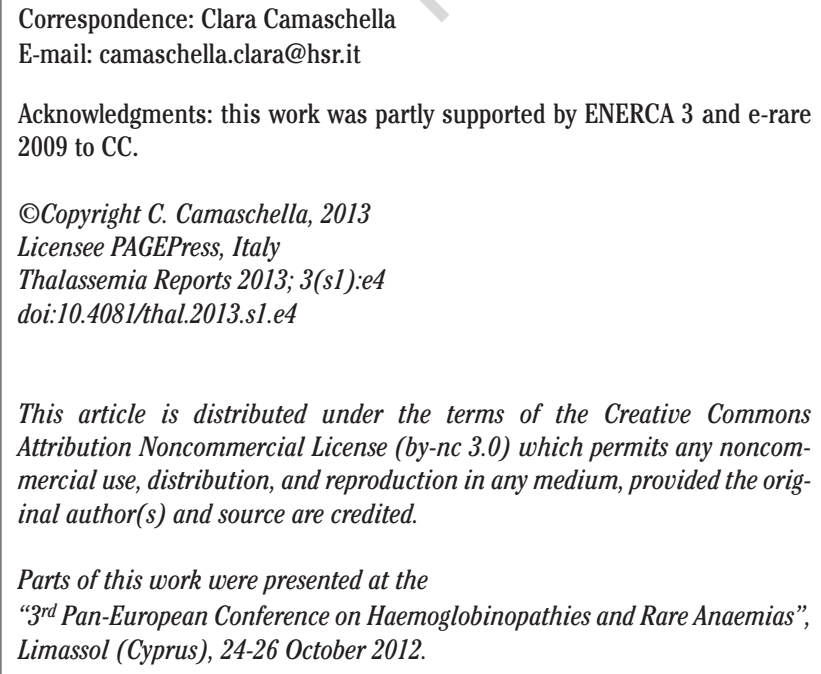

This article is distributed under the terms of the Creative Commons Attribution Noncommercial License (by-nc 3.0) which permits any noncommercial use, distribution, and reproduction in any medium, provided the original author(s) and source are credited.

Parts of this work were presented at the

"3rd Pan-European Conference on Haemoglobinopathies and Rare Anaemias", Limassol (Cyprus), 24-26 October 2012.

thesis and thus results in heme deficiency. Two disorders affect iron/sulfur cluster biogenesis: deficiency of the ATP-binding cassette B7 (ABCB7) causes X-linked sideroblastic anemia/ataxia, likely impairing the activity of ferrochelatase, which is an iron/sulfur-cluster-dependent enzyme. A recessive form affects GLRX5, a protein involved in the iron/sulfur cluster biogenesis. Aceruloplasminemia is a rare recessive syndrome characterized by anemia, diabetes, retinal degeneration, ataxia and other neurological symptoms, low serum iron but high serum ferritin, due to decreased iron recycling from macrophages and other cells. The study of these rare conditions has greatly contributed to our understanding of iron transport, utilization and recycling. Their distinction is clinically essential in order to plan the best treatment.

\section{Introduction}

Recent advances in the biology of iron metabolism and its systemic regulation have improved our understanding of iron-related disorders, identified new genetic diseases and offered novel iron related molecules/pathways to be targeted by innovative therapeutic strategies.

Iron disorders traditionally include genetic iron overload (hereditary hemochromatosis) and inherited iron-related microcytic anemias. However, some conditions characterized by primary iron overload may manifest mild anemia and some genetic iron-related anemias are accompanied by iron overload, making the clinical distinction in some cases difficult. In addition, in a more extensive classification iron disorders may also include acquired forms of secondary iron overload, as the iron loading anemias, whose prototype is beta-thalassemia (1). Another acquired iron-related disorder is represented by anemia of chronic diseases (ACD) a common anemia related to inflammatory cytokines and immune dysregulation, in which total body iron is preserved but maldistributed, with increased iron stores and iron restricted erythropoiesis. Table 1 reports a broad classification of iron disorders.

\section{Hereditary hemochromatosis}

Autosomal recessive iron overload includes the classic HFE-related hemochromatosis (type I), juvenile hemochromatosis, (type 2), due to hepcidin or hemojuvelin mutations, and TfR2-related (type 3) hemochromatosis (2). All these disorders, although with variable severity, are characterized by high transferrin saturation and high serum ferritin, iron accumulation in liver and, in severe cases, in other organs (pancreas, heart, endocrine glands) and are responsive to phlebotomy, which may prevent iron toxicity and organ failure. The prototype of these disorders and is the homozygous condition for HFE C282Y mutation that leads to an adult form of iron overload prevalently in the liver and is the most common form. The study of these conditions and of the corresponding animal models has been extremely informative to understand the regulation of systemic iron homeosta- 
sis, based on the hepatic hormone hepcidin. This is a small (25 amino acid) peptide produced by liver that regulates the surface expression of the sole known cell iron exporter, ferroportin and thus regulates the iron release to plasma (3). It is now clear from human and animal studies that all recessive hemochromatosis are disorders in which hepcidin production is insufficient compared to iron stores. These disorders are a model for iron overload that occurs in the iron loading anemia, exemplified by untransfused beta-thalassemia intermedia.

Autosomal dominant hemochromatosis, also called 'ferroportin disease', is due to mutations of the SLC40A1 gene, encoding ferroportin and is characterized in most cases by iron loading of Kupffer cells, normal transferrin saturation and high serum ferritin. Few mutations of SLC40Al cause hepcidin resistance and lead to a phenotype indistinguishable from hemochromatosis. Recessive hemochromatosis is not characterized by anemia; instead, patients show high tolerance towards repeated phlebotomies. On the contrary, some patients with ferroportin disease show marginal anemia or phlebotomy intolerance, indicating a disorder borderline between iron overload and iron deficient erythropoiesis. The issue of reduced tolerance to phlebotomy should be taken into account when planning iron depletion therapy in ferroportin disease.

\section{Inherited iron-related anemias}

Rare inherited anemias may affect the hepcidin pathway and thus iron absorption, or iron transport and utilization by the erythron and macrophage iron recycling. All these disorders are characterized by microcytic-hypochromic erythrocytes because of reduction of heme/hemoglobin formation.

\section{Iron-refractory iron-deficiency anaemia (IRIDA)}

Among the inherited anemias due to abnormal iron metabolism one of the most important, likely the most common, is the recessive Ironrefractory iron-deficiency anemia (IRIDA), due to mutations of TMPRSS6. The disease was first described in the Mask mouse, which shows high hepcidin levels and iron deficiency, unresponsive to oral iron. This mouse allowed the cloning of the Tmprss6, the gene that encodes matriptase 2, a transmembrane serine protease that suppresses hepcidin production in vivo (4). The Mask mouse was shown to have a homozygous deletion of the serine protease domain of Tmprss 6 and has the same phenotype of the mouse with the homozygous deletion of the Tmprss6 gene (Tmprss6 null mouse)(5). Several patients have been described homozygous or compound heterozygous for TMPRSS6 mutations, that affect not only the serine protease, but also other protein structural domains $(6,7)$. They are usually children or adults with longstanding anemia, show mild-moderate degree of anemia, but extremely low MCV, MCH and transferrin saturation, while serum ferritin levels may be within the normal range or only slightly decreased. Anemia seems not to be present at birth but develops in the first months of life. Hepcidin serum levels are especially high considering that in iron deficiency hepcidin levels are usually low/undetectable. Since hepcidin blocks ferroportin export activity in duodenal cells, patients are resistant to oral and only partially responsive to parenteral iron. The latter finding is explained by a less efficient iron release from macrophages since ferroportin is degraded by the inappropriately high hepcidin levels.

Other forms of anemia are due to decreased iron transport or erythroblast iron uptake. These disorders include deficiency of the iron carrier transferrin (Tf) and of proteins of the Transferrin Receptor (TfR) cycle and are characterized by anemia and iron overload without

Table 1. Classification of inherited iron-related disorders

\begin{tabular}{lccc} 
HEREDITARY HEMOCHROMATIOSIS & Gene & Inheritance & \multicolumn{1}{c}{ Phenotype } \\
Type 1 (classic form) & HFE & AR & Iron overload (liver, other organs) slowly progressive \\
Type 2 (Juvenile form) & HAMP, HJV & AR & Iron overload (liver, other organs) early onset, rapidly progressive \\
\hline Type 3 & TFR2 & AR & Iron overload (liver, other organs) early onset, slowly progressive \\
Ferroportin disease & FPN & AD & Iron overload (macrophages) \\
\hline Type 4 Hemochromatosis & FPN & AD & Iron overload (liver, other organs) \\
\hline & & &
\end{tabular}

Defective iron absorption

IRIDA TMPRSS6 AR A A $\quad$ Microcytic anemia. Iron deficiency

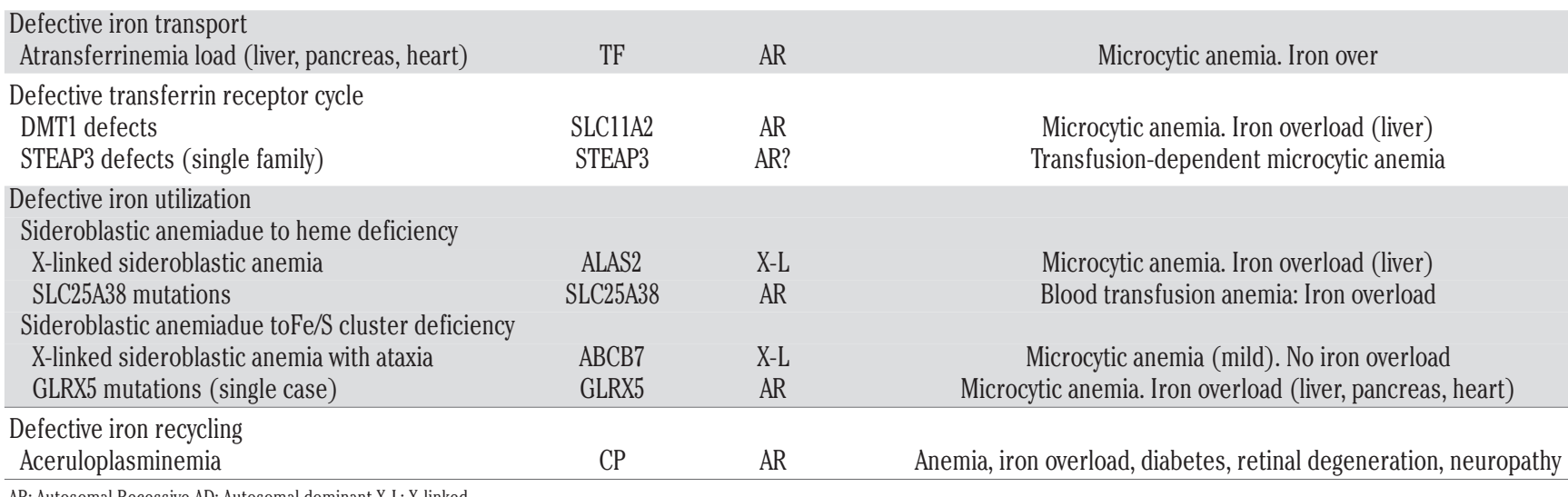

AR: Autosomal Recessive AD: Autosomal dominant X-L: X-linked 
the typical features of sideroblastic anemia. They include atransferrinemia and molecular defects of divalent metal transporter 1 (DMT1) and 6 -transmembrane epithelial antigen of the prostate 3 (STEAP3).

\section{Atransferrinemia}

Atransferrinemia (or hypotransferrinemia) is a genetic recessive anemia, extremely rare, well known since the last century. It is the prototype of disorders of TfR cycle because of deficiency of the transferrin receptor ligand. It presents with severe anemia and iron overload that involves several tissues, including liver, pancreas and heart. Hypotransferrinemia must be treated by plasma (or transferrin) infusions, a treatment that allows survival and normal development and corrects anemia. Stimulating the erythropoietic activity infused transferrin reduces iron overload because iron is mobilized from stores to erythropoiesis. In addition hepcidin production is increased after transferrin (plasma) infusions (8).

\section{DMT1 and STEAP3 mutations}

Defects of TfR1 are not reported in patients and homozygous deletion of TfR1 is lethal in murine embryos. However, patients are known with mutations of DMT1 (9) and of STEAP3 (10), a reductase involved in transferrin receptor cycle. All these patients are extremely rare: less than 10 families are known with DMT1 mutations and only a single family is known with STEAP3 deficiency. Patients have microcytic anemia of variable severity, usually less severe than hypotransferrinemia, high transferrin saturation and high serum ferritin, and, as occurs in iron loading anemia, have insufficient hepcidin production. Patients with DMT1 mutations respond to erythrocyte stimulating agents (ESA) increasing the hemoglobin levels of 1-2 g, but without changing the red cell size (9). The three siblings with STEAP3 defects have a combination of a missense mutation and a hypomorphic allele. They have severe, transfusion-dependent anemia.

\section{Sideroblastic anemia}

Inherited sideroblastic anemia includes several forms of anemias due to defective erythroblast iron utilization and characterized by iron accumulation in mitochondria. The classification of sideroblastic and non-sideroblastic anemia takes relies on the presence of ringed sideroblasts (more than 15\%) in the bone marrow erythroblasts after Perl's staining. Sideroblastic anemia is characterized by reduced iron utilization in the mitochondria that commonly leads to decreased heme synthesis and more rarely to reduced iron-sulfur cluster biogenesis (11). The classification of sideroblastic anemia is detailed in Table 1. The commonest form is X-linked, affects prevalently males and is due to delta-amino-levulinic-synthase-2-acid (ALAS2) mutations. This form is important because patients may respond to pharmacological doses of pyridoxine (vitamin B6)(12). The recessive form, affects the SLC25A38 gene, which encodes a protein belonging to the family of transporters of the mitochondrial inner membrane, considered a potential importer of glycine, an amino acid essential for delta-amino-levulinic-acid (ALA) synthesis in the heme biosynthetic pathway. SLC25A38-related sideroblastic anemia is usually more severe than the ALAS2-related disease, affects both genders and is a transfusion-dependent condition since infancy (12). Two sideroblastic forms of anemia affect iron/sulfur cluster biogenesis, the second pathway of mitochondrial iron utilization in the cell. The first is the deficiency of the ATP-binding cassette B7 (ABCB7), which causes a syndromic form, called X-linked sideroblastic anemia with ataxia. This defect causes a mild anemia, never accompanied by iron overload, but a form of non-progressive cerebellar ataxia (13). It is likely that the defect impairs the activity of ferrochelatase, an iron/sulfur-cluster-dependent enzyme, not only in erythroid precursors but also in neuronal cells. A recessive sideroblastic anemia, so far reported in a single patient, affects GLRX5, a protein involved in the iron/sulfur cluster biogenesis (14). In all forms of sideroblastic anemia independently of the type, iron is rapidly sequestered in mitochondria, making the cell cytosol iron deficient. This induces an increased iron uptake through the increased expression of TfR and causes systemic iron overload through the activation of the erythroid regulator and suppression of hepcidin activity. In some patients with sideroblastic anemia patients iron depletion induced by the use of iron chelation (or by phlebotomy combined with pyridoxine supplementation in ALAS2 defects) may increase the hemoglobin levels (14). Thus iron chelation is indicated in all forms with iron loading either primary of secondary to blood transfusions.

\section{Aceruloplasminemia}

Aceruloplasminemia is a rare recessive syndrome, due to mutations of the $\mathrm{CP}$ gene that encodes the plasma protein ceruloplasmin and is a represents an inherited defect of iron recycling. Ceruloplasmin has ferroxidase activity and cooperates with ferroportin to export iron to circulating transferrin. This is especially relevant in macrophages, hepatocytes and neuronal cells. Patients may show mild anemia, low transferrin saturation, low serum copper, undetectable ceruloplasmin. They show also signs of iron overload with high serum ferritin and increased iron storage in liver, pancreas and basal ganglia. The clinical triad of retinal degeneration, diabetes mellitus and neurologic disease appears only in midlife, making early diagnosis complex. Liver iron overload can be demonstrated by magnetic resonance imaging (MRI) (15) or liver biopsy. MRI of the brain reveals iron accumulation in basal ganglia. Since the disease is extremely rare treatment experience is limited. Experimental approaches are based on low dose iron chelators that cross the bloodbrain barrier, as in other neurodegenerative disorders (16).

\section{Acquired iron related disorders}

Untransfused beta-thalassemia is the prototype of Iron loading anemias, because a huge ineffective erythropoiesis in this condition drives increased iron absorption and release from macrophage operating on hepcidin suppression. Indeed whereas after transfusion hepcidin levels increase in thalassemic patients they are low/undetectable in thalassemia intermedia in basal conditions due to transcriptional suppression likely operated by hypoxia and by the negative signal form the expanded erythropoiesis, a signal that should also be present in iron deficiency. One potential candidate to this effect is GDF15, although its real role is still debated (3).

ACD is an acquired disorder that occurs in chronic infections, immune dysregulation and cancer. It is characterized by relative deficiency of erythropoietin, decreased bone marrow response to anemia and iron maldistribution with increased iron stores especially in macrophages and hepatocytes and decreased iron in the bone marrow at the erythropoietic (17) level. The pathophysiology of the disease and of iron abnormalities relies on excessive cytokine production, especially Il6 and Il1beta. It is a common form of mild.-moderate anemia, characterized by high hepcidin production. Treatment is addressed against the primary disorder, although some patients may respond to i.v. iron, or erythropoietin.

\section{Diagnostic approaches to rare iron-related anemias}

The precise diagnosis of rare iron related anemias requires family studies, measurement of all iron parameters, the peripheral blood 
smears and, in selected cases, bone marrow examination after Perl's staining. In cases with iron overload the evaluation of tissue (liver) iron is mandatory either using magnetic resonance imaging (MRI) or, in selected cases, liver biopsy. A first distinction should be done between conditions with increased transferrin saturation and increased serum ferritin (signs of iron overload) and those with low transferrin saturation, as IRIDA (7). In the first group are included sideroblastic and non sideroblastic conditions. To diagnose sideroblastic anemia requires examination of the bone marrow smear after Perl's staining for the search of ringed sideroblasts. They could be seen also in peripheral blood smear as coarse iron inclusions (Pappenheimer bodies). In sideroblastic anemia search for mutations by DNA analysis may be less relevant than in other cases. Differential diagnosis especially when dealing with adult patients is with the acquired forms. Non sideroblastic forms are related to defective uptake of iron by the erythron and due to DMT1, STEAP3 or other proteins of the TfR cycle mutations.

Except for atransferrinemia and aceruloplasminemia all these cases require DNA sequencing of the correspondent genes. Second level tests include soluble transferrin receptor, which is a sign of expanded erythropoiesis and serum hepcidin dosage (18) in the case of IRIDA. In most cases of IRIDA nucleotide sequencing of TMPRSS6 gene may provide the precise diagnosis. For these reasons index cases should be referred for second level diagnosis to selected expert centers.

\section{Differential diagnosis}

Rare genetic anemias with iron overload need to be differentiated by other congenital microcytic anemia as thalassemia, whereas IRIDA implies a differential diagnosis with anemia of chronic diseases (ACD). The mechanism of iron loading is similar in all iron loading anemias and is due to the expanded erythropoiesis that drives iron absorption through hepcidin suppression. However, the defective globin chain synthesis of beta- and alpha-thalassemia, especially thalassemia intermedia may be easily recognized based on family studies, hemoglobin and/or gene analysis.

IRIDA may be differentiated by ACD that show common features, because of high hepcidin production secondary to cytokines stimulation. If the inflammatory disorder is evident distinction is easy; in other cases the determination of the CRP level may help to exclude inflammatory clinically silent conditions. Usually microcytosis and hypochromia are absent/borderline and transferrin saturation is less decreased unless ACD is longstanding, especially severe or is accompanied by true iron deficiency.

\section{Treatment}

Different approaches are required according to the defects. Atransferrinemia requires regular plasma infusions. DMT1 deficiency has been treated with erythropoietin in cases of severe anemia in order to avoid transfusions. Sideroblastic anemia due to ALAS2 mutations are worth to be treated with pyridoxine, although not all cases are responsive. The other forms of sideroblastic anemia are not responsive to B6. Few cases of severe sideroblastic anemia due to SLC25A38 have received a allogeneic bone marrow transplantation. Severe cases require blood transfusions and iron chelation treatment. The latter approach has been reported to ameliorate the degree of anemia in few patients. There is not established treatment of aceruloplasminemia: iron chelation may worsen anemia but for the limited experience available cannot reverse neurological symptoms.

Animal models are available for most of the described disorders. New therapeutic strategies are under development to manipulate the hepcidin pathway in order to reduce excessive hepcidin production (e.g. in IRIDA and ACD) or to increase hepcidin activity (hemochromatosis and thalassemia)(19). These approaches are aimed either to contrast the protein effect or interfere with protein synthesis in the first case and to substitute the missing hormone or increase its production in the second case. A change of our approaches to treatment of the described disorders may be foreseen in the near future

\section{References}

1. Andrews NC. (2008) Forging a field: the golden age of iron biology. Blood, 112, 219-230.

2. Camaschella C. (2005) Understanding iron homeostasis through genetic analysis of hemochromatosis and related disorders.Blood. 106(12):3710-7.

3. Hentze MW, Muckenthaler MU, Galy B, Camaschella C. (2010) Two to tango: regulation of Mammalian iron metabolism. Cell. 142:2438.

4. Du X, She E, Gelbart T, Truksa J, Lee P, Xia Y, Khovananth K, Mudd S, Mann N, Moresco EM, Beutler E, Beutler B. (2008) The serine protease TMPRSS6 is required to sense iron deficiency. Science. 320(5879):1088-92.

5. Folgueras AR, de Lara FM, Pendás AM, Garabaya C, Rodríguez F, Astudillo A, Bernal T, Cabanillas R, López-Otín C, Velasco G. (2008) Membrane-bound serine protease matriptase-2 (Tmprss6) is an essential regulator of iron homeostasis. Blood. 112:2539-45.

6. Finberg KE. (2009) Iron-refractory iron deficiency anemia. Semin Hematol.;46(4):378-8.

7. Camaschella C (2012) State of the Art - How I manage patients with atypical microcytic anaemia. Br J Haematol. in press.

8. Trombini P, Coliva T, Nemeth E, Mariani R, Ganz T, Biondi A, Piperno A. (2007) Effects of plasma transfusion on hepcidin production in human congenital hypotransferrinemia. Haematologica. 92(10):1407-10.

9. Grandchamp B, Hetet G, Kannengiesser C, Oudin C, Beaumont C, Rodrigues-Ferreira S, Amson R, Telerman A, Nielsen P, Kohne E, Balser C, Heimpel H.(2011) A novel type of congenital hypochromic anemia associated with a nonsense mutation in the STEAP3/TSAP6 gene. Blood. 118:6660-6.

10. Iolascon A, Camaschella C, Pospisilova D, Piscopo, C, Tchernia, G \& Beaumont C. (2008) Natural history of recessive inheritance of DMT1 mutations. The Journal of pediatrics. 152, 136-139.

11. Camaschella $C$ and Poggiali E. (2011) Inherited disorders of iron metabolism. Current opinion in pediatrics, 23, 14-20.

12. Fleming MD. (2011) Congenital sideroblastic anemias: iron and heme lost in mitochondrial translation. Hematology/the Education Program of the American Society of Hematology. American Society of Hematology. Education Program, 525-531.

13. Camaschella C. (2008) Recent advances in the understanding of inherited sideroblastic anaemia. British journal haematol., 143, 2738.

14. Camaschella C, Campanella A, De Falco L, Boschetto L, Merlini R, Silvestri L, Levi S, Iolascon A. (2007) The human counterpart of zebrafish shiraz shows sideroblastic-like microcytic anemia and iron overload. Blood. 110(4):1353-8.

15. St Pierre TG, Clark PR, Chua-anusorn W, Fleming AJ, Jeffrey GP, Olynyk JK, Pootrakul P, Robins E and Lindeman R. (2005) Noninvasive measurement and imaging of liver iron concentrations using proton magnetic resonance. Blood. 105, 855-861.

16. Abbruzzese G, Cossu G, Balocco M, Marchese R, Murgia D, Melis M, Galanello R, Barella S, Matta G, Ruffinengo U, Bonuccelli U, Forni 
GL. (2011) A pilot trial of deferiprone for neurodegeneration with brain iron accumulation. Haematologica. 96:1708-11.

17. Goodnough LT, Nemeth E and Ganz T. (2010) Detection, evaluation, and management of iron-restricted erythropoiesis. Blood, 116, 4754-4761.
18. Ganz T, Olbina G, Girelli D, Nemeth E and Westerman M. (2008) Immunoassay for human serum hepcidin. Blood, 112, 4292-4297.

19. Ganz T, Nemeth E. (2011) The hepcidin-ferroportin system as a therapeutic target in anemias and iron overload disorders. Hematology Am Soc Hematol Educ Program. 2011:538-42.

\section{Oral presentation}

Oral presentation is available online 